\title{
Riemann-Hilbert problem associated with the vector Lakshmanan-Porsezian-Daniel model in the birefringent optical fibers
}

Beibei $\mathrm{Hu}^{1}$, Ji Lin ${ }^{1}$, and Ling Zhang ${ }^{2}$

${ }^{1}$ Zhejiang Normal University

${ }^{2}$ Chuzhou University

September 29, 2021

\begin{abstract}
In this paper, we investigate vector Lakshmanan-Porsezian-Daniel (VLPD) model which can be used to describe the ultrashort pulses in the birefringent optical fiber. Based on the unified transformation method, the Riemann-Hilbert problem is introduced and initial-boundary value problems of the VLPD model are studied. By solving the formulated matrix Riemann-Hilbert problem, the potential function solutions of the VLPD model can be reconstructed. Moreover, that the spectral functions are not independent but meet the so-called global relation is shown.
\end{abstract}

\section{Hosted file}

VLPD RHP.pdf available at https://authorea.com/users/438414/articles/539616-riemannhilbert-problem-associated-with-the-vector-lakshmanan-porsezian-daniel-model-in-thebirefringent-optical-fibers

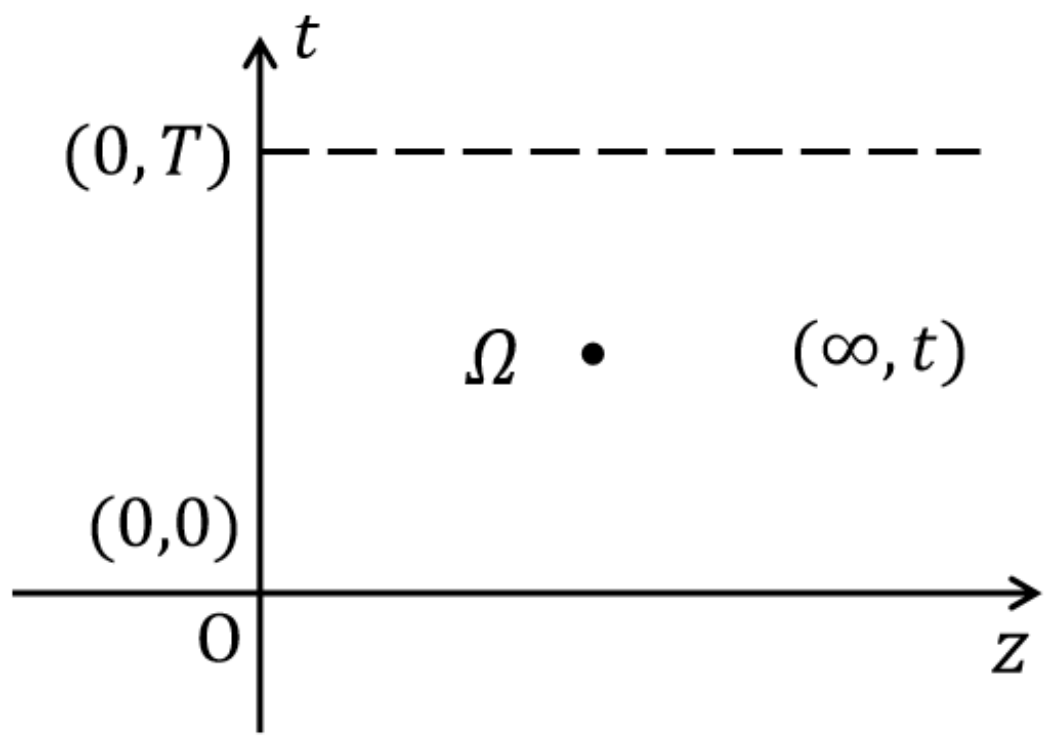



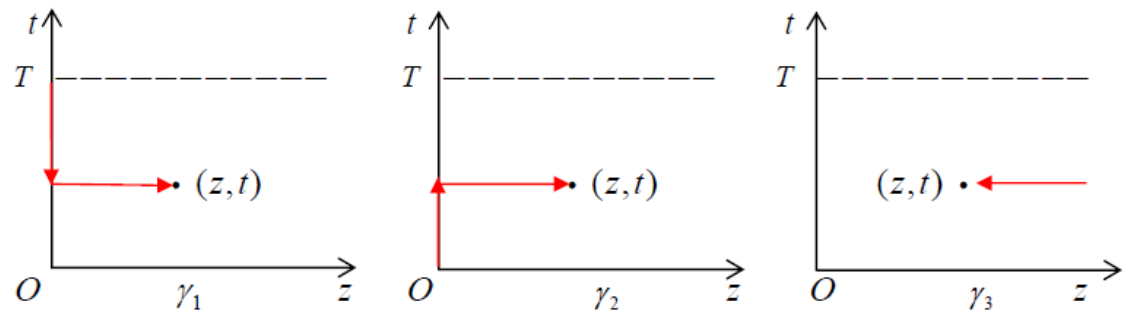